\title{
Stay the course
}

\author{
Key stakeholders, journals and funders must enable the additional research and early data sharing needed to \\ advance the adoptive T cell therapy field as a whole.
}

n March, after reviewing adverse reactions associated with their trials of CD19-targeted chimeric antigen receptor (CAR) T cell therapy of hematological malignancies, Memorial Sloan Kettering Cancer Center (MSKCC) voluntarily paused the trials and proposed trial amendments to the US Food and Drug Administration (FDA). Although the FDA reviewed the amendments and released the hold after six weeks, news of the halt nevertheless prompted expressions of doubt in the general therapeutic strategy of administering patient $\mathrm{T}$ cells that have been engineered to express a CAR or T cell receptor (TCR) specific for an antigen on the tumor.

But rather than questioning the overall approach or holding it to a higher standard than more conventional cancer therapies, the focus should be on enabling the research needed to predict, prevent and treat the toxicities associated with this type of treatment. The extent and durability of the therapeutic responses observed thus far, albeit in the relatively small pool of patients who have been treated, demand persistence.

The toxicities observed in patients treated in anti-CD19 CAR trials range from fever to hypotension to neurological symptoms. As they seem to be the result of cytokines released by the engineered T cells and other leukocyte populations, they are collectively called cytokine release syndrome (CRS). But because we lack a complete understanding of which cytokines are responsible for which symptoms, it's not currently possible to reliably prevent or treat all aspects of CRS. It's also very difficult to predict which patients will develop the most severe forms of CRS. This is important because although most symptoms have been transient, some symptoms have resolved with minimal intervention, whereas others required a stay in intensive care. This variability is perhaps not surprising in light of potential patient-to-patient heterogeneity in terms of prior treatments, conditioning regimens, tumor burdens and engineered $\mathrm{T}$ cell preparations.

This is not to say that the field is not making progress. Recently reported correlations between severe CRS and serum C-reactive protein concentrations or pretreatment tumor burden may have predictive value, and interleukin-6 receptor blockade was effective in several cases. With regard to prevention, several approaches are being explored, including reducing the dose of infused T cells (a step taken by MSKCC), injecting T cells of more defined subtype or differentiation state and equipping $\mathrm{T}$ cells with inducible suicide genes.

But much more needs to be done. In the face of relatively small trial cohorts thus far, and patient-to-patient as well as trial-to-trial variability, pooling and sharing of data may be key. Especially if these data include systematic analysis of blood cytokine profiles and thorough descriptions of the doses and phenotypes of T cells administered, these pooled data sets may make it easier to spot generalizable trends and correlations between particular cytokines and CRS symptoms, for example. Journals can play a role here, too, by encouraging thorough reporting of patient pretreatment characteristics, toxicity data, and treatment protocols, either in the journal publication itself or in databases like ClinicalTrials.gov. That said, it is important that the recent influx of commercial interest in this field, and the competitive concerns that inevitably come with it, do not hinder early data sharing and publication if early data sharing and publication can forward the field as a whole.

In addition to clinical data sharing, preclinical models of CRS would be an enormous asset. Unfortunately, no animal models of severe CRS have been described, at least none able to recapitulate the toxicities seen in anti-CD19 CAR T cell therapy. Funding agencies should prioritize these efforts, which may not be trivial, as a new mouse model may be needed to model the toxicities associated with adoptive $\mathrm{T}$ cell therapy of each type of malignancy.

And safely extending the application of adoptive T cell therapy beyond CD19-expressing malignancies is another challenge. CD19 is ideal because it is a surface protein-and therefore potentially targetable by a one-sizefits-all CAR - and because among healthy cells it is expressed only on B cells which, even if killed by anti-CD19 CAR T cells, can be functionally replaced to some extent by intravenous immunoglobulin. It is proving difficult to identify similar targets on other malignancies, as tumor targets are often expressed on essential healthy cells. The importance of this issue cannot be underestimated, as illustrated by recent incidents where engineered $\mathrm{T}$ cells recognized peptides on the brain or heart.

But here again the field is making progress, for example by targeting mutated peptides. Although this approach was recently applied to induce prolonged stabilization of disease in a patient with metastatic cholangiocarcinoma, because each tumor bears unique mutations, interpatient heterogeneity — and even intrapatient heterogeneity in a patient bearing multiple tumor nodules-would again come into play with this approach. And it's not certain that every tumor will bear even one mutation suitable for targeting by a TCR or CAR. Nevertheless, especially in light of the general trend towards personalized cancer therapy, with exome sequencing of patient tumors becoming more and more routine, this strategy should not be prematurely abandoned. Involving computational biology communities to develop user-friendly approaches for mining sequencing data for peptides likely to be targetable by TCRs, and engineering streamlined approaches for cloning TCRs able to recognize these peptides, would help move this strategy into standard clinical use.

Of course, finding a suitable target antigen may not be the only challenge facing efforts to treat solid tumors. It may be necessary to engineer additional functionalities into $\mathrm{T}$ cells to render them capable of resisting the immunosuppressive environments characteristic of some solid malignances. Here, again, additional basic research funding will be essential.

It's clear that the obstacles facing this field are not trivial. But neither is the promise shown by this therapy. Thorough characterization, publication and presentation of its toxicities, as well as its successes, will benefit the field as a whole. Journals can help enable this sort of reporting, and commercial interests must not hold it back. 